\title{
Morphofunctional Plasticity in the Adult Hypothalamus Induces Regulation of Polysialic Acid-Neural Cell Adhesion Molecule through Changing Activity and Expression Levels of Polysialyltransferases
}

\author{
Sylvia Soares, Ysander von Boxberg, Michèle Ravaille-Veron, Jean-Didier Vincent, and Fatiha Nothias \\ Institut Alfred Fessard, Centre National de la Recherche Scientifique UPR 2212, Gif sur Yvette, 91198, France
}

Polysialic acid-neural cell adhesion molecule (PSA-NCAM) expression in the adult nervous system is restricted to regions retaining a capacity for morphological plasticity. For the female rat hypothalamoneurohypophysial system (HNS), we have previously shown that lactation induces a dramatic decrease in PSA-NCAM, while leaving the level of total NCAM protein unchanged. Here, we wanted to elucidate the molecular mechanisms leading to a downregulation of PSA, thereby stabilizing newly established synapses and neurohemal contacts that accompany the increased activity of oxytocinergic neurons.

First, we show that the overall specific activity of polysialyltransferases present in tissue extracts from supraoptic nuclei decreases by $\sim 50 \%$ during lactation. So far, two polysialyltransferase enzymes, STX and PST, have been characterized for their capacity to transfer PSA onto NCAM in vitro. Using a competitive RT-PCR on RNA extracts from the HNS, we demonstrate furthermore a significant decrease in the expression levels of both STX and PST mRNAs in lactating versus virgin animals. Interestingly, this downregulation of NCAM polysialylation is not correlated with the post-transcriptional regulation of variable alternative spliced exon splicing, in contrast to neural development. The control of polysialylation via a regulation of both enzyme activity and expression underlines the important role of this post-translational modification of NCAM in morphofunctional plasticity in adult brain.

Key words: PSA-NCAM; PST; STX; competitive RT-PCR; enzymatic activity; lactation
Development of the nervous system, and its structural remodeling in the adult, rely on site- and time-dependent expression of specific combinations of adhesion molecules. In addition, adhesive properties of individual cell adhesion molecules can be modified both on post-transcriptional and post-translational levels. For the neural cell adhesion molecule (NCAM), addition of polysialic acid (PSA) to the fifth Ig-like domain facilitates events such as cell migration, neurite growth, and synaptic reorganization (for review, see Rutishauser and Jessell, 1988; Rougon, 1993; Rutishauser, 1993; Seki and Arai, 1993; Fryer and Hockfield, 1996). Postnatal loss of PSA is generally associated with stabilization of NCAM-mediated cell-cell interactions and synapse formation (Szele et al., 1994).

PSA is a large, negatively charged homopolymer of $\alpha-2,8$ sialic acid. Two characterized polysialyltransferases, STX (ST8SiaII) and PST (ST8SiaIV) (Livingston and Paulson, 1993; Nakayama et al., 1995), have been shown to add PSA onto NCAM in vitro; however, there is no direct evidence of the exact role of these enzymes in vivo. Highly expressed during development, their expression is downregulated with maturation of the nervous system, in close correlation with PSA-NCAM expression (Kuro-

\footnotetext{
Received Nov. 4, 1999; revised Jan. 10, 2000; accepted Jan. 14, 2000.
}

This work was supported by Centre National de la Recherche Scientifique and European Community Grant BM-H4-CT95-0524. We thank Dr. Alain Prochiantz for critical reading of this manuscript, Dr. Geneviève Rougon for providing us with PSA-NCAM antibodies (anti-Men-B) and EndoN enzyme, and Dr. Pat Doherty and Dr. Jane Saffell for the NCAM-Fc chimera. We also thank Stéphane Père and Jean-Paul Bouillot for technical help.

Correspondence should be addressed to Fatiha Nothias, Institut Alfred FessardCentre National de la Recherche Scientifique, 1 Avenue de la Terrasse, 91198 Gif sur Yvette, France. E-mail: nothias@iaf.cnrs-gif.fr.

Copyright (๑) 2000 Society for Neuroscience 0270-6474/00/202551-07\$15.00/0 sawa et al., 1997; Wood et al., 1997; Hildebrandt et al., 1998b; Ong et al., 1998). STX and PST messenger expression persists, however, in restricted areas of the adult brain (Kurosawa et al., 1997; Phillips et al., 1997), i.e., in the olfactory system, and in certain areas capable of structural reorganization, such as the hippocampus and, in particular, the hypothalamoneurohypophysial system (HNS).

The HNS, comprising the hypothalamic magnocellular nuclei [supraoptic nuclei (SON) and paraventricular nuclei (PVN)] that project into the neurohypophysis $(\mathrm{NH})$, undergoes specific morphological modifications in association with certain physiological changes, e.g., prolonged dehydration and lactation (Hatton, 1990). In the hypothalamus of a lactating female, the astrocytic coverage of oxytocinergic neurons is reduced, thereby increasing membrane appositions and synaptic contacts, and in the $\mathrm{NH}$, the interactions of oxytocinergic axon terminals with blood vessels are enhanced. This morphological state, which is reversible after weaning, is associated with increased, highly synchronous electrical activity of oxytocinergic neurons (Lincoln and Wakerley, 1975). We have shown previously that during lactation, subsequent to the observed morphofunctional changes in the HNS, PSA-NCAM expression is dramatically decreased in both SON and NH. In contrast, no modifications in the total amount of NCAM protein or in its mRNA were observed. The HNS may therefore represent a good in vivo model for morphological plasticity affecting the polysialylation of NCAM.

To elucidate the molecular mechanisms underlying the changes in PSA-NCAM expression, we evaluated the overall activity of polysialyltransferases in the HNS of virgin and lactating females. We also analyzed, using a competitive RT-PCR, any potential 
lactation-induced changes in expression in mRNAs of both STX and PST. Finally, we analyzed the regulation of NCAM expression on a post-translational level by examining the alternative splicing of the $30 \mathrm{bp}$ variable alternative spliced exon (VASE) on NCAM mRNA. In contrast to PSA, VASE peptide presence on the fourth NCAM Ig-like domain, which is developmentally regulated, appears to reduce neurite outgrowth and enhance NCAM-mediated adhesion (Small et al., 1988).

\section{MATERIALS AND METHODS}

Animals. Virgin adult and lactating (at $10 \mathrm{~d}$ of lactation) Wistar rats (IFFA Credo, L'Arbresle, France) were used. For enzyme activity tests and RT-PCR, animals were killed by decapitation, and the brains and neurohypophysis were quickly removed, frozen with isopentane at $-40^{\circ} \mathrm{C}$ and stored at $-80^{\circ} \mathrm{C}$. SON were dissected by punching them out from frozen brain slices.

Immunohistochemistry. Animals were prepared for brain sectioning as described (Nothias et al., 1997). Coronal sections $(30 \mu \mathrm{m})$ were incubated with primary antibody directed against polysialic acid [IgM antibody, anti-Men-B (Rougon et al., 1986), followed by peroxidase-labeled secondary antibody (Cappel/Flobio)]. Peroxidase reactivity was revealed with $0.05 \%$ diaminobenzidine tetrahydrochloride and $0.01 \%$ hydrogen peroxide in $0.05 \mathrm{~m}$ Tris buffer, $\mathrm{pH}$ 7.6. Care was taken to treat sections from virgin and lactating rats in parallel under exactly the same conditions. To visualize both cell bodies and fibers, adjacent sections were treated by Kluver and Barrera staining.

Polysialyltransferase activity test. From SON of virgin and lactating rats, a protein extract enriched for polysialyltransferases was prepared according to Oka et al. (1995). The enzyme activity test reaction was performed in a final volume of $50 \mu \mathrm{l}$, containing $10 \mu \mathrm{g}$ purified NCAM-Fc chimera (generously provided by P. Doherty, Guy's Hospital, London, UK) (Saffell et al., 1997), $20 \mathrm{~mm} \mathrm{CMP-}{ }^{14} \mathrm{C}$-neuraminic acid $\left(2.8 \times 10^{5} \mathrm{cpm}\right)$, $10 \mathrm{~mm} 2-\mathrm{N}$-morpholino ethanesulfonic acid (MES), $20 \mathrm{~mm} \mathrm{MnCl}_{2}, 2.5$ mM ATP, and the enzyme-enriched cell extract. After incubation for $3 \mathrm{hr}$ at $37^{\circ} \mathrm{C}$, the reaction was terminated by addition of EDTA $(50 \mathrm{~mm})$. One-half of the sample was digested with EndoN (generously provided by G. Rougon, CNRS Luminy, Marseille, France) for $4 \mathrm{hr}$ at $37^{\circ} \mathrm{C}$. Treated and untreated samples were then spotted onto Whatman GF/C paper disks, rinsed three times in MES buffer $(20 \mathrm{mM})$ containing $\mathrm{NaCl}$ $250 \mathrm{~mm}$, rinsed once with ethanol $95 \%$, and air-dried. Radioactivity on the disks was measured using a scintillation counter, and the difference between EndoN-treated and untreated samples was taken as a measure of the amount of sialic acid incorporated. Total protein concentration was determined by the Lowry method, and specific enzymatic activity was calculated and compared for each case using an ANOVA test.

Quantification of STX and PST $m R N A$ s by competitive RT-PCR. Total RNA was extracted with Triaxis reagent according to the manufacturer's specification (Genaxis) and treated with DNase $(0.02 \mathrm{U} / \mu \mathrm{l}$; Promega). To clone STX and PST, $5 \mu \mathrm{g}$ aliquots of hypothalamic RNA were incubated for $5 \mathrm{~min}$ at $70^{\circ} \mathrm{C}$ with $1 \mu \mathrm{g}$ of random and $1 \mu \mathrm{g}$ of oligo-dT primers in a final volume of $25 \mu \mathrm{l}$, and reverse transcription was performed with Moloney murine leukemia virus-RT (Promega) according to the manufacturer's recommendations. For selective amplification of STX and PST, primers were carefully chosen from nonoverlapping sequences. The primer sequence for STX was from mouse (GenBank accession number X83562; 5'-GGG TCT TGC TGA ACA GCG GC-3' and 5'GTG TAG CCA TAC TTG AGG CTG-3'; nucleotides 658-1186); the primer sequence for PST was from rat (GenBank accession number U90215; 5'-ACT GAG GAG CAC CAA GAG ACG C-3' and 5'-CCA TGA AGG CAG GAA TCC AAA GG-3'; nucleotides 31-637). PCR was performed with Tfl DNA polymerase (Promega) according to instructions of the manufacturer. After an initial step of 3 min at $93^{\circ} \mathrm{C}, 40$ cycles were performed with $1 \mathrm{~min}$ at $92^{\circ} \mathrm{C}, 1 \mathrm{~min}$ at $58^{\circ} \mathrm{C}$ for STX and $62^{\circ} \mathrm{C}$ for PST, then $1 \mathrm{~min}$ at $72^{\circ} \mathrm{C}$. Amplified products (528 bp for STX and $606 \mathrm{bp}$ for PST) were subcloned in pGEM-T Easy (Promega) plasmid, and authenticity was verified by sequencing (Genome Express) and comparison with respective GenBank data. Standard STX $\Delta$ and PST $\Delta$ were constructed by digesting STX and PST cDNA $(2 \mu \mathrm{g})$ with AvaI and Tth 111 , respectively, for $2 \mathrm{hr}$ at $37^{\circ} \mathrm{C}$, eliminating a fragment of $156 \mathrm{bp}$ for STX and $110 \mathrm{bp}$ for PST. Digested cDNAs were purified on a microspin column (Pharmacia Biotech) and ligated with T4 DNA ligase (Promega), and the size of the standards (372 bp for STX $\Delta$ and $496 \mathrm{bp}$ for PST $\Delta$ ) was verified on an agarose gel, after amplification by PCR using the same primers as above. Plasmids were then linearized with SalI and transcribed into cRNA by T7 RNA polymerase.

For the competitive PCR, varying amounts (from 0.5 to $0.1 \mathrm{pg}$ for STX and from 10 to $1 \mathrm{pg}$ for PST) of the internal standard cRNAs were added to a constant amount ( $2 \mu \mathrm{g}$ for PST and $5 \mu \mathrm{g}$ for STX) of total RNA, extracted from virgin or lactating SON and $\mathrm{NH}$. Each mixture was reverse-transcribed, and $2 \mu \mathrm{l}$ of the product was subjected to PCR as described above, except that primers were $5^{\prime}$-radiolabeled with $\gamma^{-32} \mathrm{P}$ ATP. Briefly, $1 \mathrm{nmol}$ of each primer was incubated for $30 \mathrm{~min}$ at $37^{\circ} \mathrm{C}$ with $50 \mu \mathrm{Ci}$ of $\gamma_{-}{ }^{32} \mathrm{P}$-ATP and T4-polynucleotide kinase $(20 \mathrm{U}$; Promega) in the appropriate buffer in $50 \mu \mathrm{l}$ final volume. For the radioactive PCR ( $25 \mu$ l final volume), each template was used in duplicate for either 35 or 40 cycles. Seven microliters of PCR products were loaded on an $8 \%$ polyacrylamide gel, run at $150 \mathrm{~V}$, dried, and exposed on a phosphorimaging screen (PhosphorImager, Molecular Dynamics) for further quantification. A competitive PCR linear regression curve was obtained by plotting the ratio of radioactivity [expressed as $\log (\mathrm{cpm})]$ incorporated into the respective amplification products of target mRNA and cRNA internal standards against the quantities of internal standard used in each reaction. The relative amounts of STX and PST target RNAs present in the different tissues were directly calculated from this curve and reported to the total amount of RNA used in each RT-PCR (Liu et al., 1997).

Semiquantitative RT-PCR of NCAM-VASE mRNAs. $\gamma$ - ${ }^{32} \mathrm{P}-\mathrm{ATP}$ radiolabeled oligonucleotides $5^{\prime}$-ACC TGG AGA ACG TCC ACC CGA AAC ATC-3' and 5'-AGG ACA CAC GAG CAT GGC TGC GTA CCA CCA-3' covering both flanking regions of the VASE sequence were chosen as primers for amplification of NCAM mRNAs with or without the VASE exon (Small and Akeson, 1990). Hot PCR was performed on SON and NH cDNA from virgin and lactating (at 1 and $10 \mathrm{~d}$ ) rats. An initial step of $3 \mathrm{~min}$ at $93^{\circ} \mathrm{C}$ was followed by $1 \mathrm{~min}$ at $92^{\circ} \mathrm{C}, 1 \mathrm{~min}$ at $55^{\circ} \mathrm{C}$, then $1 \mathrm{~min}$ at $72^{\circ} \mathrm{C}$ for extension, for 25,30 , and 35 cycles. Seven microliters of each reaction product were loaded on a $12 \%$ polyacrylamide gel that was run at $150 \mathrm{~V}$, dried, and exposed to a phosphorimaging screen (PhosphorImager; Molecular Dynamics) for quantification. The PCR reaction gives rise to two bands, the top corresponding to $\mathrm{NCAM} \mathrm{VASE}^{+}$mRNA (120 bp) and the bottom (90 bp) to NCAMVASE $^{-}$mRNA. Data are presented as the ratio of radioactivity [ex-

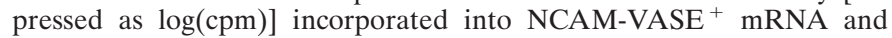
NCAM-VASE ${ }^{-}$mRNA and compared for virgin versus lactating conditions using an ANOVA test.

\section{RESULTS}

We have recently shown that the morphof unctional modifications in the hypothalamus during lactation are accompanied by a significant decrease in PSA-NCAM, an isoform of NCAM strongly expressed by the SON of virgin females (Nothias et al., 1997). This decrease in PSA staining becomes obvious at the end of the first week of lactation and is most prominent by day 10 (Fig. 1b,c) [see also Nothias et al. (1997)], whereas the levels of total NCAM protein remain unchanged. Here, we demonstrate that the PVN exhibit the same progressive decline in PSA-NCAM staining (Fig. $1 d, e)$. It is noteworthy that in contrast to other hypothalamic nuclei, this change in PSA-NCAM during lactation is exclusively observed in the magnocellular nuclei (SON and PVN) that project into the $\mathrm{NH}$.

This decrease in PSA immunoreactivity during lactation could in principle be caused by a change in the specific activity of polysialyltransferases, a change in the expression level of sialyltransferase mRNAs, or both. In all experiments that follow, virgin rats were always compared with animals at day 10 of lactation, the time point at which the decrease in PSA levels is most obvious (see above).

To analyze the overall polysialyltransferase activity, polysialyltransferase-enriched tissue extracts were prepared from the SON of virgin and lactating rats (Oka et al., 1995), and enzymatic activity was determined by the incorporation of CMP${ }^{14} \mathrm{C}$-sialic acid into NCAM-Fc chimeras. As shown in Figure 2, SON extracts of lactating rats exhibited a specific polysialyltransferase activity that was reduced by $\sim 50 \%$ in comparison to that 

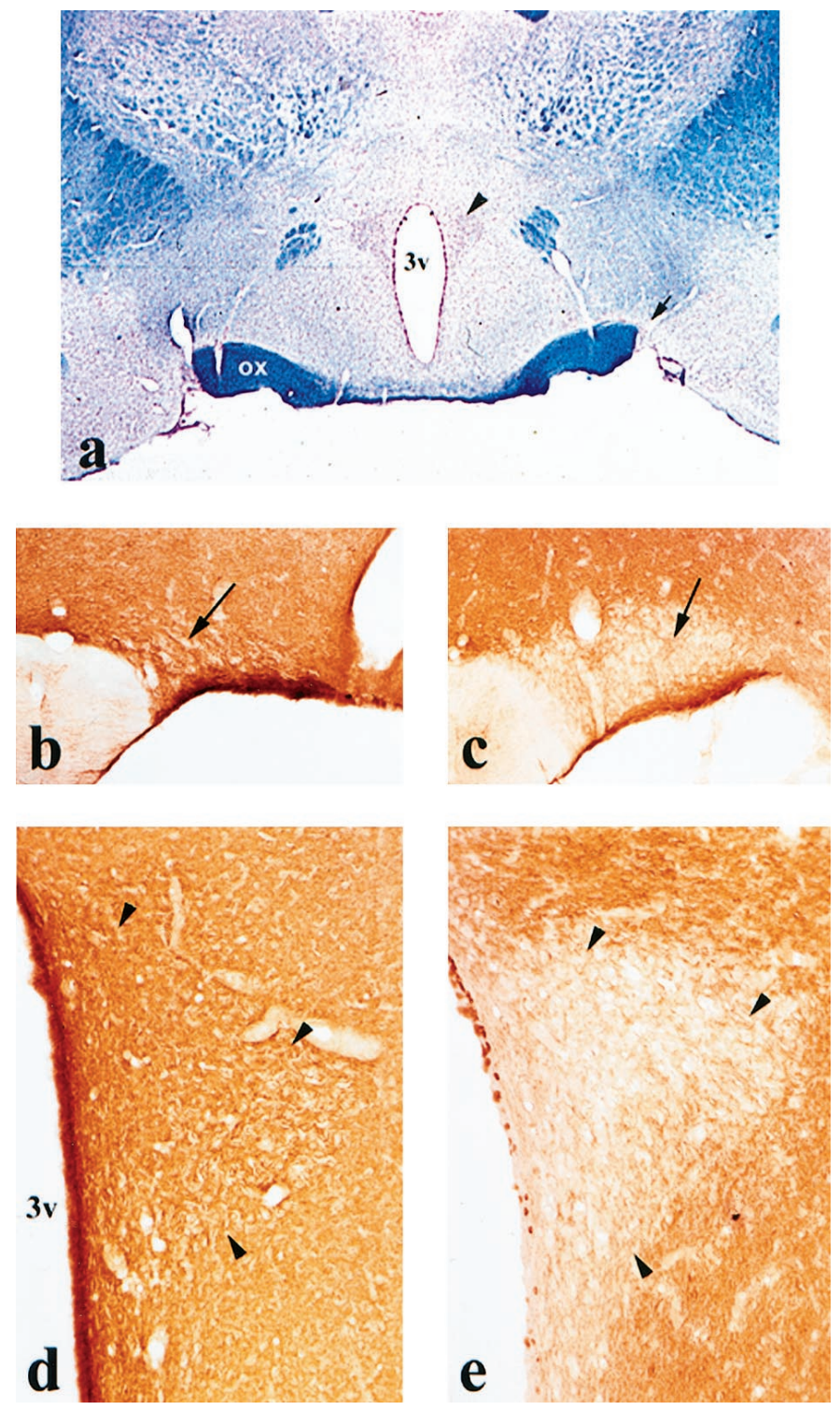

Figure 1. PSA-NCAM expression in SON and PVN of virgin and lactating rats. $a$, Photomicrograph of a coronal section from adult rat brain, at the level of SON (arrow) and PVN (arrowhead) hypothalamic nuclei; Kluver and Barrera staining. $b-e$, Photomicrographs of PSA-NCAM immunostaining in SON $(b, c)$ and PVN $(d, e)$ nuclei of virgin $(b, d)$ and $10 \mathrm{~d}$ lactating $(c, e)$ rats. During lactation, polysialic acid immunoreactivity is dramatically decreased in both nuclei, whereas the rest of the hypothalamus remains highly stained. Magnifications: $a, 17 \times ; b, 67 \times ; c$, $80 \times ; d-e, 120 \times$.

of nonlactating rats. Similar values were obtained for the PVN (data not shown). Thus, the observed decrease of PSA-NCAM in both hypothalamic nuclei during lactation appears to be closely correlated with a specific downregulation of polysialyltransferase activity.

The reduction in specific enzymatic activity observed in the SON during lactation (Fig. 2) could be attributable, at least in part, to a downregulation of enzyme expression itself. Thus, we have analyzed the relative mRNA expression levels of the two well characterized vertebrate polysialyltransferases STX and PST in SON and NH of virgin and lactating animals. With respect to the limited amounts of RNA to be extracted from these tissues, a competitive RT-PCR appeared to be the adequate approach for

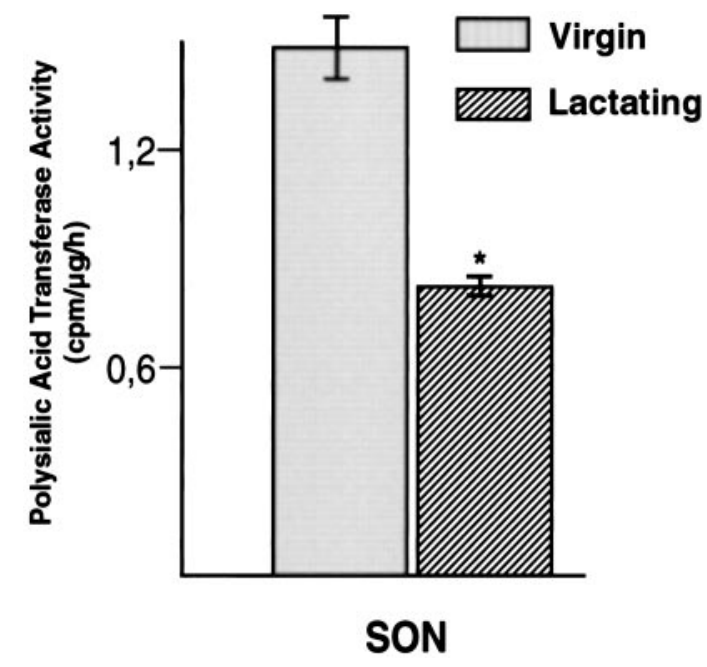

Figure 2. Decrease in overall polysialyltransferase activity in the SON during lactation. Polysialyltransferase-enriched tissue extracts obtained from virgin and $10 \mathrm{~d}$ lactating animals were used to catalyze incorporation of ${ }^{14} \mathrm{C}$-labeled sialic acid into PSA chains on NCAM-Fc chimeras of a given concentration. The radioactivity incorporated under each condition, in relation to the total protein content of the corresponding tissue extract, was taken as a measure for the specific enzyme activity $\left({ }^{*} p<0.05\right.$; ANOVA test).

quantification. By carefully choosing nonoverlapping regions from the known coding sequences for both enzymes, we amplified a 528 bp stretch of STX and a 606 bp stretch of PST cDNA, from which corresponding internal standards were constructed (372 bp for STX $\Delta$ and $496 \mathrm{bp}$ for PST $\Delta$ ), to be used as competitors in the RT-PCR.

Consistent with previous in situ hybridization data (Phillips et al., 1997), electrophoretic analysis of the competitive RT-PCR reaction products confirmed the presence of mRNA transcripts encoding for STX (Fig. 3) and PST (Fig. 4) in adult SON, as well as in PVN (data not shown) and NH. Incorporation of radioactivity into the target RNA amplification products (top band) depended on the relative amount of competing internal standard cRNAs (bottom band). Quantification was performed by calculating the ratio between the log of the cpm of the two bands (STX/STX $\Delta$ or PST/PST $\Delta$ ) across the series of concentrations of internal standards. For both STX (three independent experiments) and PST (four independent experiments), the point of equivalence [i.e., the ratio $\log (\operatorname{cpm~STX}) / \log (\operatorname{cpm~STX} \Delta)=1]$ is obtained at a concentration of internal standards that differs considerably between virgin versus lactation conditions. Indeed, in the case of STX (see graph in Fig. 3), the equivalence point corresponds to $\sim 0.5 \mathrm{pg}$ of STX $\Delta$ for the SON of virgin and $\sim 0.2$ $\mathrm{pg}$ for that of lactating rats. The content in STX mRNA per microgram of total RNA is $\sim 0.1 \mathrm{pg}$ for virgin SON and decreases by $60 \%$ to $\sim 0.04 \mathrm{pg}$ during lactation. For PST mRNA expression (see graph in Fig. 4), the point of equivalence lies at $\sim 4.5 \mathrm{pg}$ of PST $\Delta$ for the SON of virgin rats and $\sim 3 \mathrm{pg}$ for the SON of lactating rats. This corresponds to a decrease of $35 \%$, from $\sim 2.3$ pg PST mRNA per microgram total RNA for the SON of a virgin rat to $\sim 1.5 \mathrm{pg}$ of a lactating one. Thus, in the SON, mRNAs encoding for PST are more abundant than those encoding for STX. Most important, however, these data demonstrate that downregulation of the expression of both polysialyltransferase mRNAs is at least one of the molecular mechanisms involved in the decrease of polysialylation of NCAM during lactation. 

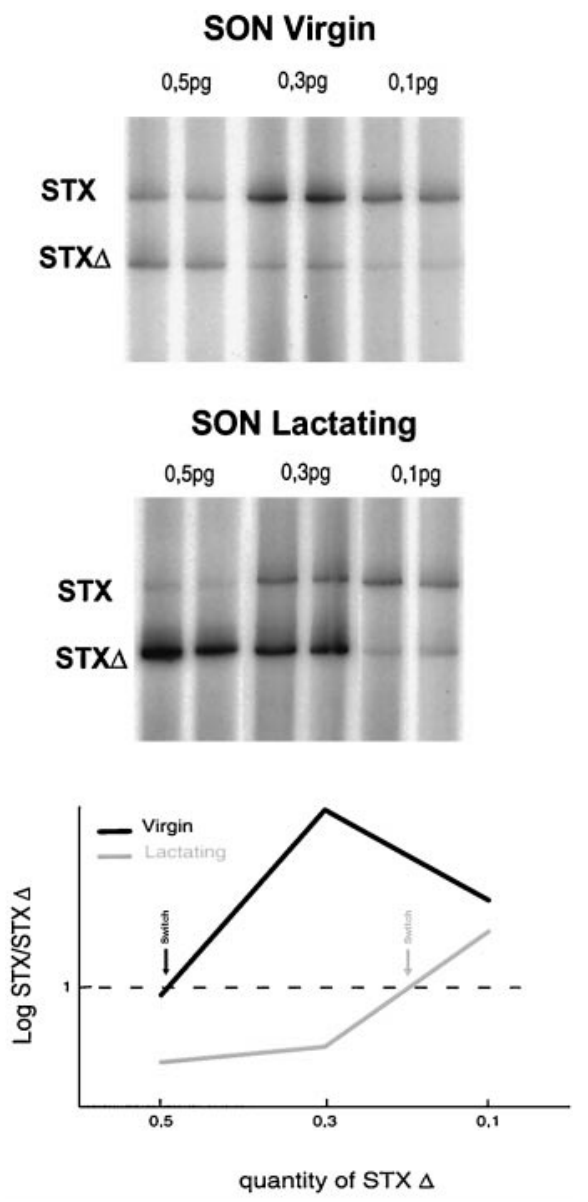

Figure 3. STX expression in the SON of virgin and lactating rats. Top, Total RNA was extracted from the SON of virgin rats and at day 10 of lactation. Competitive RT-PCRs were performed using $\gamma-{ }^{32} \mathrm{P}$-ATPlabeled STX-specific primers with different amounts of STX $\Delta$ internal standard cRNA $(0.5,0.3$, and $0.1 \mathrm{pg})$ and a constant amount of total SON RNA $(5 \mu \mathrm{g})$. The reaction products were separated on an acrylamide gel, and the radioactivity incorporated into each band was determined using a Phosphorimager. Graph, Phosphorimager quantitative analysis of the above gels: to evaluate the relative STX mRNA levels in virgin and lactating SON, a competitive RT-PCR linear regression curve is constructed by plotting for each concentration of internal standard cRNA, and the ratios of $\log (\mathrm{cpm})$ are incorporated into STX versus STX $\Delta$ reaction products. The point of equivalence $[\log (\mathrm{cpm}$ STX $) / \log (\mathrm{cpm}$ $\operatorname{STX} \Delta)=1$; indicated by arrows] switches from $0.5 \mathrm{pg}$ of STX $\Delta$ for virgin SON to $0.2 \mathrm{pg}$ for lactating SON, corresponding to a decrease of $60 \%$.

A similar competitive RT-PCR analysis was also performed for the $\mathrm{NH}$. In contrast to the SON, however, we did not detect any changes in STX and PST mRNA expression during lactation (data not shown), although PSA levels are decreasing in the $\mathrm{NH}$ (Nothias et al., 1997). Furthermore, the expression of PST mRNA in the $\mathrm{NH}$ was comparatively low, whereas the amount of STX mRNA was as high as in SON. For the SON and the NH, results of the competitive RT-PCR experiments are summarized in Table 1.

In parallel, we wanted to determine whether lactation would affect NCAM expression in the HNS, not only on the posttranslational but also on the post-transcriptional level. In particular, we have studied the expression of VASE on NCAM in SON and $\mathrm{NH}$ isolated from virgin or lactating rats. VASE is a $30 \mathrm{bp}$ spliced exon that codes for a 10 amino acid peptide in the fourth Ig-like domain on a certain percentage of NCAM molecules,
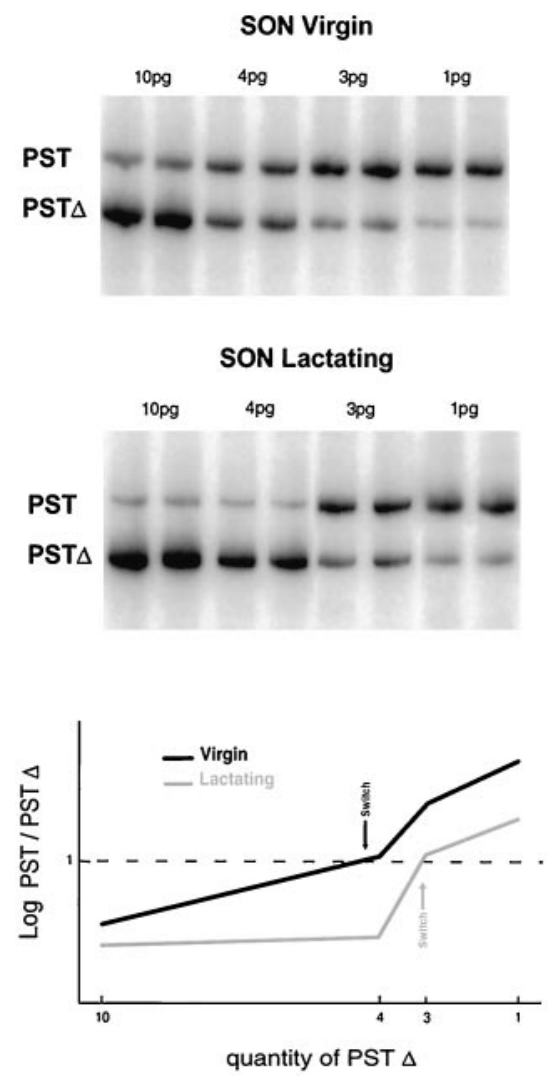

Figure 4. PST expression in the SON of virgin and lactating rats. Gels, The radioactive RT-PCR was performed as detailed in Figure 3, using PST-specific primers with different amounts of PST $\Delta$ internal standard cRNA $(10,4,3$, and $1 \mathrm{pg})$ and $2 \mu \mathrm{g}$ of total SON RNA. Graph, Phosphorimager quantitative analysis of the above gels. Competitive RT-PCR linear regression plot of $\log (\mathrm{cpm}$ PST $) / \log (\mathrm{cpm}$ PST $\Delta)$ against the concentrations of internal standard used in each experiment. The point of equivalence $[\log (\mathrm{cpm}$ PST $) / \log (\mathrm{cpm} \operatorname{PST} \Delta)=1$; indicated by arrows $]$ switches from $4.5 \mathrm{pg}$ for the virgin SON to $3 \mathrm{pg}$ for lactating SON, corresponding to a decrease in PST mRNA levels of $35 \%$.

depending on the tissue and the state of maturation. By semiquantitative RT-PCR, we compared the relative expression levels of mRNAs encoding for NCAM with (NCAM-VASE ${ }^{+}$) or without (NCAM-VASE ${ }^{-}$) the VASE exon. Primers were chosen to co-amplify mRNAs containing or not containing the VASE exon (Small and Akeson, 1990), yielding a 120 and a 90 bp PCR amplification product, respectively (Fig. 5).

For the neurohypophysis of both virgin and lactating rats, PCR amplification products corresponded only to the bottom band, indicating that the $\mathrm{NH}$ expresses uniquely NCAM lacking the VASE exon. In the hypothalamic nuclei, however, NCAM mRNAs with and without the VASE exon are present. As shown in Figure 5 for the SON (similar results were obtained for the PVN; data not shown), the NCAM-VASE ${ }^{+} / \mathrm{NCAM} \mathrm{VASE}^{-}$ratio in virgin versus lactating rats is slightly decreased, but in a reproducible manner, during lactation. This decrease is observed from the first day of lactation (data not shown). Thus, in addition to the post-translational regulation of its polysialylation, NCAM expression is regulated also on the post-transcriptional level. In contrast to the downregulation of polysialylation, however, VASE exon splicing occurs at the beginning of the lactation-induced morphological remodeling. 
Table 1. Competitive RT-PCR analysis of STX and PST mRNA expression in SON and NH of virgin and lactating rats

\begin{tabular}{|c|c|c|c|c|c|c|}
\hline \multirow[b]{2}{*}{ Tissue extracts } & \multicolumn{3}{|l|}{ STX mRNA } & \multicolumn{3}{|l|}{ PST mRNA } \\
\hline & $\begin{array}{l}\text { Point of } \\
\text { equivalence }\end{array}$ & $\begin{array}{l}\text { (pg mRNA/ } / \mu \mathrm{g} \\
\text { total RNA) }\end{array}$ & & $\begin{array}{l}\text { Point of } \\
\text { equivalence }\end{array}$ & $\begin{array}{l}(\mathrm{pg} \text { mRNA/ } / \mu \mathrm{g} \\
\text { total RNA) }\end{array}$ & \\
\hline SON virgin & $0.5 \mathrm{pg}$ & 0.1 & & $4.5 \mathrm{pg}$ & 2.3 & \\
\hline SON lactating & $0.2 \mathrm{pg}$ & 0.04 & Decrease of $60 \%$ & $3 \mathrm{pg}$ & 1.5 & Decrease of $35 \%$ \\
\hline $\mathrm{NH}$ virgin & $\sim 0.4 \mathrm{pg}$ & $\sim 0.1$ & & $\sim 0.8 \mathrm{pg}$ & $\sim 0.4$ & \\
\hline NH lactating & id & id & No change & id & id & No change \\
\hline
\end{tabular}

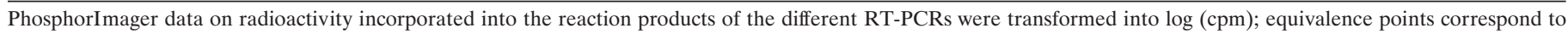

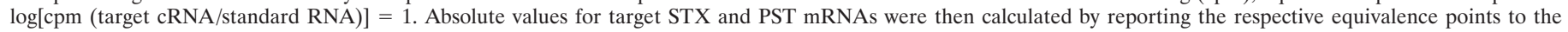
amount of total RNA used in each reaction. See also Figures 3 and 4. id, Identical.

\section{DISCUSSION}

The present study demonstrates for the first time a clear link between the modulation of PSA levels on the NCAM molecule, coinciding with morphological plasticity in the adult brain, and the regulation of gene expression for the STX and PST polysialyltransferases. The change in STX and PST mRNA levels may be responsible in part for the observed decrease in the overall activity of polysialyltransferases in the hypothalamic magnocellular nuclei. However, similar changes in PSA levels in the neurohypophysis during lactation are not accompanied by changing STX and PST gene expression levels.

Among the six sialyltransferases characterized, only STX and PST have been found to exhibit a considerable degree of homology in their genomic structure, exceeding $60 \%$ within the catalytic domain (Eckhardt and Gerardy-Schahn, 1998). They are the only enzymes capable of polymerizing sialic acid, and they have a substrate specificity that is restricted to NCAM (Kojima et al., 1996). Despite these similarities, certain differences in their activities have also been noted: mouse STX activity is stimulated in the presence of $\mathrm{Mn}^{2+}$ or $\mathrm{Mg}^{2+}$ and that of PST is stimulated by $\mathrm{Ca}^{2+}$; the efficiency of PST to polysialylate NCAM in vitro seemed much higher than that of STX (Kojima et al., 1996; Angata et al., 1998). Furthermore, their differential expression with respect to cell type and developmental stages (Angata et al., 1997; Kurosawa et al., 1997; Phillips et al., 1997), and the analysis of genomic structure and promoter activities of the corresponding genes (Yoshida et al., 1996; Takashima et al., 1998), suggested the regulation of PST and STX by distinct transcription factors and probably also distinct signal transduction pathways (Kojima et al., 1996). Little is known about the regulation of polysialyltransferase activity. Electrical activity of neurons, or contractile activity in cultured myotubes (Rafuse and Landmesser, 1996) appears to stimulate NCAM polysialylation; this may be based on the effect of changes in intracellular $\mathrm{Ca}^{2+}$ concentrations (Bruses and Rutishauser, 1998) and/or inhibition of protein kinase C (PKC) on the activity of polysialyltransferases. Furthermore, it has recently been shown that $\mathrm{PKC} \delta$, and the $29 \mathrm{kDa}$ acidic protein 14-3-3, copurify with immunoprecipitated PST (H. Gallagher and C. M. Regan, personal communication); thus, control of PST activity may be effectuated by a mutual regulation of the activity of PKC $\delta$ by 14-3-3 protein, and vice versa.

Several comparative studies were undertaken to determine the expression pattern of PST and STX in developing and adult nervous systems. In early stages of development, PST and STX mRNAs are relatively abundant and usually coexpressed in most of the tissues examined (Phillips et al., 1997), the level of STX mRNA being several times higher than that of PST (Angata et al.,
1997). Through postnatal developmental stages, expression of STX and PST is increasingly heterogeneous: STX is substantially downregulated, to become almost undetectable in the adult, whereas the decline in PST expression is moderate (Hildebrandt et al., 1998a; Ong et al., 1998). In the present study, both polysialyltransferase mRNAs were detected in the SON and PVN, the mRNA encoding for PST being more abundant than STX mRNA, consistent with previous in situ hybridization data (Phillips et al., 1997). Apparent contradictions between the different studies concerning the relative expression levels of the STX and PST genes might be attributable to the various species and brain regions analyzed, rendering a direct comparison difficult. Nevertheless, all of these studies, including ours, agree in that the amount of PSA found in developing or adult brain is closely correlated with the levels of PST and STX mRNA (Kurosawa et al., 1997; Phillips et al., 1997; Ong et al., 1998).

In certain restricted regions of the adult brain that maintain detectable levels of PSA, such as the hypothalamic nuclei, STX and PST expression remains relatively high. Here, we demonstrate that changes in NCAM-PSA levels in both SON and PVN during lactation coincide precisely with a change in STX and PST gene expression. This suggests that the in vivo polysialylation of NCAM is at least partially regulated at the level of polysialyltransferase gene expression. Thus, these enzymes may play an important role in neuronal plasticity in the adult.

Transfection of cells in vitro with either enzyme, STX or PST, enhances NCAM-mediated neurite outgrowth in a similar way. When both PST and STX are cotransfected, synthesis of polysialic acid is more efficient than with PST or STX alone, suggesting a synergistic rather than competitive action (Angata et al., 1997, 1998). In fact, it has been shown that PST adds PSA preferentially at the sixth $N$-glycosylation site, closest to the NCAM transmembrane domain (Angata et al., 1998), which in turn may facilitate polysialylation at the fifth site by STX. Although direct in vivo evidence is still lacking, such a cooperative mechanism may well be active in the HNS system, given that PST and STX are always coexpressed in brain regions in which extensive polysialylation is required even in the adult (Phillips et al., 1997; present study).

Independently from the analysis of STX and PST mRNA expression, we also determined the overall enzymatic activity of polysialyltransferases in tissue extracts from rat SON. Thus, the observed decrease of PSA-NCAM during lactation appears to be closely related to a reduction of polysialyltransferase activity by $\sim 50 \%$. It has already been noted that in the chick, the downregulation of PSA on ciliary ganglion motoneurons during synaptogenesis precisely coincides with a decrease in polysialyltransferase activity levels (Bruses et al., 1995; Oka et al., 1995). It may 


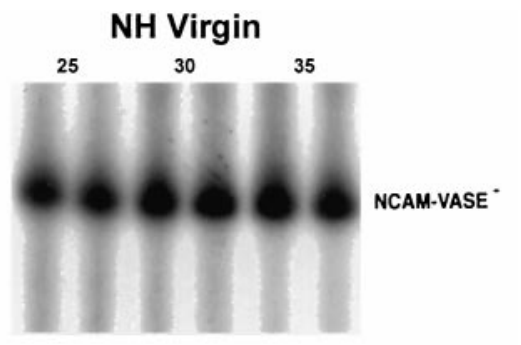

NH Lactating

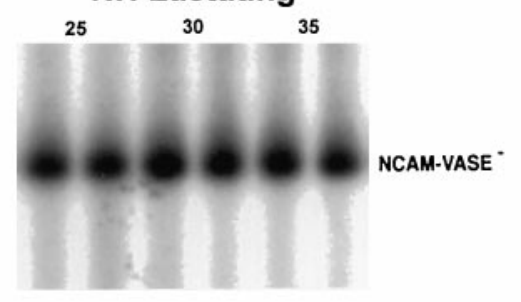

SON Virgin

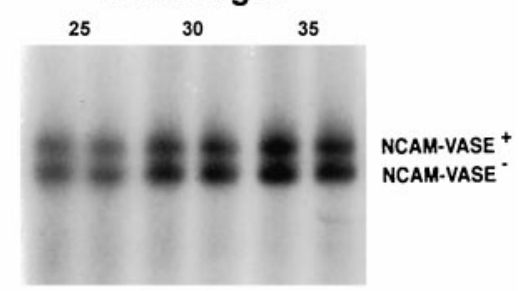

SON Lactating
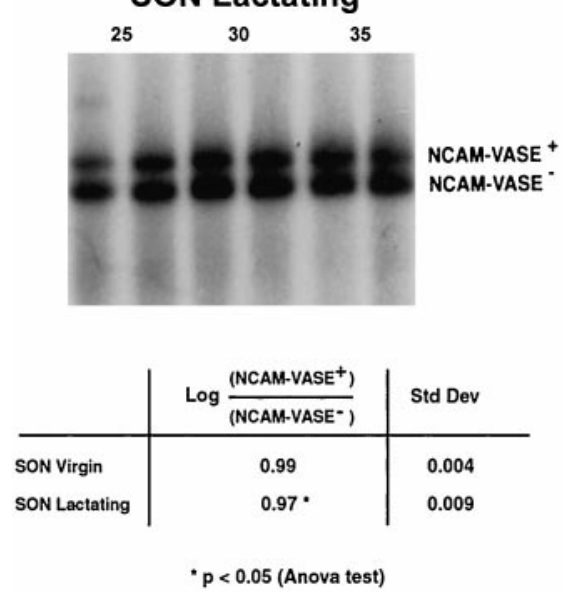

Figure 5. VASE-NCAM expression in $\mathrm{NH}$ and $\mathrm{SON}$ of virgin and lactating rats. $\gamma_{-}{ }^{32} \mathrm{P}$-ATP-labeled primers were constructed from both flanking regions of the VASE sequence to amplify a stretch of NCAM cDNA containing or not containing the VASE exon. Semiquantitative PCRs (25, 30, and 35 cycles) were performed on cDNA reversetranscribed from total RNA extracted from $\mathrm{NH}$ and SON of virgin and lactating (day 10) rats. Amplification products were separated on acrylamide gels, and the radioactivity of the bands representing NCAM$\mathrm{VASE}^{+}$(top band) and NCAM-VASE ${ }^{-}$mRNA (bottom band) was evaluated using a PhosphorImager. The table summarizes the respective values of the ratio $\log \left(\mathrm{cpm} \mathrm{NCAM} \mathrm{VASE}^{+} / \mathrm{NCAM} \mathrm{VASE}^{-}\right)$together with standard deviations. Note that although NCAM-VASE ${ }^{+}$is completely absent from NH, for both hypothalamic nuclei the ratio NCAM$\mathrm{VASE}^{+} / \mathrm{NCAM} \mathrm{VASE}^{-}$is significantly higher in virgin than in lactating rat.

be interesting that in this latter study the developmental regulation of PSA synthesis was not reflected by a change in the PST mRNA. In case of the rat SON, we present the first example of a tissue examined under two different physiological conditions in which changing polysialyltransferase activities can be correlated with changing expression levels of PST and STX genes. This does not preclude that also in the SON, specific polysialyltransferase activity could be affected independently from gene expression, as seems to be the case for the $\mathrm{NH}$. In this target organ of the oxytocinergic neurons, PSA-NCAM levels are also decreasing during the period of lactation (Nothias et al., 1997); however, we did not detect any changes in PST or STX mRNA expression. The decrease in PSA immunoreactivity in the NH may be caused by reduced PSA on the hypothalamic afferents and by a reduced specific activity of the pituicyte polysialyltransferases. Thus, as had been shown previously for chick ciliary ganglion motoneurons (Bruses and Rutishauser, 1998), the loss of PSA on NCAM in the HNS of lactating rats is not generally coupled to a decrease in polysialyltransferase mRNA expression.

Finally, adhesivity of NCAM can be modified not only on the post-translational but also, via differential splicing, on the posttranscriptional level. The alternative splicing of the $30 \mathrm{bp}$ VASE exon is developmentally regulated, and NCAM-VASE ${ }^{+}$mRNAs are highly expressed in adulthood (Small et al., 1988). Insertion of the VASE peptide increases NCAM adhesivity, and some reports have noticed a negative correlation between the presence of VASE and the degree of NCAM polysialylation (Small and Akeson, 1990; Doherty et al., 1992), at least for certain brain areas (Oka et al., 1995). Although the fourth Ig-like domain, which eventually carries the VASE peptide, has previously been implicated in NCAM polysialylation (Nelson et al., 1995), insertion of VASE into the NCAM sequence in vitro did not affect the affinity between PST and NCAM, nor PSA synthesis in general (Oka et al., 1995).

Using semiquantitative PCR, we examined a potential regulation of VASE expression in the HNS during lactation. In fact, $\mathrm{NCAM}_{-\mathrm{VASE}}{ }^{+}$and NCAM-VASE ${ }^{-}$mRNA were found in SON; the NH contained only NCAM-VASE ${ }^{-}$mRNA. Surprisingly, the downregulation of polysialylation was not paralleled by an increase in VASE expression; rather, the ratio of NCAM$\mathrm{VASE}^{+}$to NCAM-VASE ${ }^{-}$was slightly decreased in SON during lactation. In addition, this decrease was already detectable on the first day of lactation and thus seems to precede the downregulation of polysialylation. This demonstrates that VASE expression and polysialylation are two events that are controlled independently, in agreement with the findings of Oka et al. (1995). Reduced expression of the VASE peptide may well facilitate the initiation of the lactation-induced morphological changes taking place in the HNS. The new state of equilibrium that will finally be attained at $10 \mathrm{~d}$ of lactation would then be progressively stabilized by a downregulation of polysialylation.

In summary, the reversible modifications of synaptic, neuroglial, and neurohemal contacts taking place in the HNS during lactation offer an excellent possibility for studying the molecular mechanisms that are at the basis of neuronal plasticity in the adult CNS. Our results suggest that two enzymes that have been shown to polysialylate NCAM in vitro, STX and PST, may exert the same function in vivo. The lactation-induced downregulation of the degree of NCAM polysialylation in the SON may be a result, at least in part, of the observed downregulation of these two enzymes. Thus, post-translational modifications of the NCAM molecule are regulated under stringent control by at least two mechanisms, underlining the important role of a precise fine tuning of cell adhesion for plasticity of the nervous system. 


\section{REFERENCES}

Angata K, Nakayama J, Fredette B, Chong K, Ranscht B, Fukuda M (1997) Human STX polysialyltransferase forms the embryonic form of the neural cell adhesion molecule. Tissue-specific expression, neurite outgrowth, and chromosomal localization in comparison with another polysialyltransferase, PST. J Biol Chem 272:7182-7190.

Angata K, Suzuki M, Fukada M (1998) Differential and cooperative polysialylation of the neural cell adhesion molecule by two polysialyltransferases, PST and STX. J Biol Chem 273:28524-28532.

Bruses JL, Rutishauser U (1998) Regulation of neural cell adhesion molecule polysialylation to an intracellular poll of calcium. J Cell Biol 140:1177-1186.

Bruses JL, Oka S, Rutishauser U (1995) NCAM-associated polysialic acid on ciliary ganglion neurons is regulated by polysialytransferase levels and interaction with muscle. J Neurosci 15:8310-8319.

Doherty P, Moolenaar CE, Ashton SV, Michalides RJ, Walsh FS (1992) The VASE exon downregulates the neurite growth-promoting activity of NCAM 140. Nature 356:791-793.

Eckhardt M, Gerardy-Schahn R (1998) Genomic organization of the murine polysialyltransferase gene ST8SiaIV (PST-1). Glycobiology $8: 1165-1172$

Fryer HJ, Hockfield S (1996) The role of polysialic acid and other carbohydrate polymers in neural structural plasticity. Curr Opin Neurobiol 6:113-118.

Hatton GI (1990) Emerging concepts of structure-function dynamics in adult brain: the hypothalamo-neurohypophysial system. Prog Neurobiol 34:437-504.

Hildebrandt H, Becker C, Gluer S, Rosner H, Gerardy-Schahn R, Rahmann H (1998a) Polysialic acid on neural cell adhesion molecule correlates with expression of polysialyltransferases and promotes neuroblastoma cell growth. Cancer Res 58:779-784.

Hildebrandt H, Becker C, Murau M, Gerardy-Schahn R, Rahmann H (1998b) Heterogeneous expression of the polysialyltransferases ST8SiaII and ST8SiaIV during postnatal rat brain development. J Neurochem 71:2339-2348.

Kojima N, Tachida Y, Yoshida Y, Tsuji S (1996) Characterization of mouse ST8SiaII (STX) as a neural cell adhesion molecule-specific polysialic acid synthase. J Biol Chem 271:19457-19463.

Kurosawa N, Yoshida Y, Kojima N, Tsuji S (1997) Polysialic acid synthase (ST8Sia II/STX) mRNA expression in developing mouse central nervous system. J Neurochem 69:494-503.

Lincoln DW, Wakerley JB (1975) Neurosecretory activation in the rat: correlation of the suckling stimulus with the pulsatile release of oxytocin. J Physiol (Lond) 245:42P-43P.

Liu J, Morrow AL, Devaud L, Grayson DR, Lauder JM (1997) GABA receptors mediate trophic effects of GABA on embryonic brainstem monoamine neurons in vitro. J Neurosci 17:2420-2428.

Livingston BD, Paulson JC (1993) Polymerase chain reaction cloning of a developmentally regulated member of the sialyltransferase gene family. J Biol Chem 268:11504-11507.

Nakayama J, Fukuda MN, Fredette B, Ranscht B, Fukuda M (1995) Expression cloning of a human polysialyltransferase that forms the polysialylated neural cell adhesion molecule present in embryonic brain. Proc Natl Acad Sci USA 92:7031-7035.

Nelson RW, Bates PA, Rutishauser U (1995) Protein determinants for specific polysialylation of the neural cell adhesion molecule. J Biol Chem 270:17171-17179.

Nothias F, Vernier P, von Boxberg Y, Mirman S, Vincent JD (1997) Modulation of NCAM polysialylation is associated with morphofunctional modifications in the hypothalamo-neurohypophysial system during lactation. Eur J Neurosci 9:1553-1565.

Oka S, Bruses JL, Nelson RW, Rutishauser U (1995) Properties and developmental regulation of polysialyltransferase activity in the chicken embryo brain. J Biol Chem 270:19357-19363.

Ong E, Nakayama J, Angata K, Reyes L, Katsuyama T, Arai Y, Fukuda M (1998) Developmental regulation of polysialic acid synthesis in mouse directed by two polysialyltransferases, PST and STX. Glycobiology 8:415-424.

Phillips GR, Krushel LA, Crossin KL (1997) Developmental expression of two rat sialyltransferases that modify the neural cell adhesion molecule, N-CAM. Brain Res Dev Brain Res 102:143-155.

Rafuse VF, Landmesser L (1996) Contractile activity regulates isoform expression and polysialylation of NCAM in cultured myotubes: involvement of $\mathrm{Ca}^{2+}$ and protein kinase C. J Cell Biol 132:969-983.

Rougon G (1993) Structure, metabolism and cell biology of polysialic acids. Eur J Cell Biol 61:197-207.

Rougon G, Dubois C, Buckley N, Magnani JL, Zollinger W (1986) A monoclonal antibody against meningococcus group $\mathrm{B}$ polysaccharides distinguishes embryonic from adult N-CAM. J Cell Biol 103:2429-2437.

Rutishauser U (1993) Regulation of cell-cell interactions by NCAM and its polysialic acid moiety. In: Polysialic acid (Roth J, Rutishauser U, Troy II FA, eds), pp 215-227. Basel: Birkhauser Verlag.

Rutishauser U, Jessell TM (1988) Cell adhesion molecules in vertebrate neural development. Physiol Rev 68:819-857.

Saffell J, Williams E, Mason I, Walsh F, Doherty P (1997) Expression of a dominant negative FGF receptor inhibits axonal growth and FGF receptor phosphorylation stimulated by CAMs. Neuron 18:231-242.

Seki T, Arai Y (1993) Distribution and possible roles of the highly polysialylated neural cell adhesion molecule (NCAM-H) in the developing and adult central nervous system. Neurosci Res 17:265-290.

Small SJ, Akeson R (1990) Expression of the unique NCAM VASE exon is independently regulated in distinct tissues during development. J Cell Biol 111:2089-2096.

Small SJ, Haines SL, Akeson RA (1988) Polypeptide variation in an N-CAM extracellular immunoglobulin-like fold is developmentally regulated through alternative splicing. Neuron 1:1007-1017.

Szele FG, Dowling JJ, Gonzales C, Theveniau M, Rougon G, Chesselet MF (1994) Pattern of expression of highly polysialylated neural cell adhesion molecule in the developing and adult rat striatum. Neuroscience 60:133-144.

Takashima S, Yoshida Y, Kanematsu T, Kojima N, Tsuji S (1998) Genomic structure and promoter activity of the mouse polysialic acid synthase (mST8SiaIV/PST) gene. J Biol Chem 273:7675-7683.

Wood GK, Liang JJ, Flores G, Ahmad S, Quirion R, Srivastava LK (1997) Cloning and in situ hybridization analysis of the expression of polysialyltransferase mRNA in the developing and adult rat brain. Brain Res Mol Brain Res 51:69-81.

Yoshida Y, Kurosawas N, Kanematsu T, Kojima N, Tsuji S (1996) Genomic structure and promoter activity of the mouse polysialic acid synthase gene (mST8SiaII). J Biol Chem 271:30167-30173. 